\title{
Lie Algebras with Nilpotent Length Greater than that of each of their Subalgebras
}

\author{
David A. Towers ${ }^{1}$
}

Received: 24 August 2016 / Accepted: 27 November 2016 / Published online: 16 December 2016

(C) The Author(s) 2016. This article is published with open access at Springerlink.com

\begin{abstract}
The main purpose of this paper is to study the finite-dimensional solvable Lie algebras described in its title, which we call minimal non- $\mathcal{N}$. To facilitate this we investigate solvable Lie algebras of nilpotent length $k$, and of nilpotent length $\leq k$, and extreme Lie algebras, which have the property that their nilpotent length is equal to the number of conjugacy classes of maximal subalgebras. We characterise the minimal non- $\mathcal{N}$ Lie algebras in which every nilpotent subalgebra is abelian, and those of solvability index $\leq 3$.
\end{abstract}

Keywords Lie algebras · Solvable $\cdot$ Nilpotent series $\cdot$ Nilpotent length $\cdot$ Chief factor · Extreme $\cdot$ Nilregular $\cdot$ Characteristic ideal $\cdot A$-algebra

Mathematics Subject Classification (2010) 17B05 $\cdot$ 17B20 $\cdot 17$ B30 $\cdot 17$ B50

\section{Introduction}

Let $L$ be a Lie algebra, and let $L^{(0)}=L, L^{(i+1)}=\left[L^{(i)}, L^{(i)}\right]$ be its derived series. Recall that $L$ is solvable if there exists $r$ such that $L^{(r)}=0$; the smallest such $r$ is called the derived length of $L$. Similarly, $L^{1}=L, L^{i+1}=\left[L^{i}, L\right]$ is the lower central series of $L ; L$ is nilpotent of nilpotency index $r$ if $L^{r+1}=0$ but $L^{r} \neq 0$. Throughout, $L$ will denote a finitedimensional solvable Lie algebra over a field $F$. The symbol ' $\oplus$ ' will denote an algebra direct sum, whilst ' $\dot{+}$ ' will denote a direct sum of the underlying vector space structure alone. If $U$ is a subalgebra of $L$ we define $U_{L}$, the core (with respect to $L$ ) of $U$, to be the largest ideal of $L$ contained in $U$. We say that $U$ is core-free in $L$ if $U_{L}=0$.

Presented by Yuri Drozd

David A. Towers

d.towers@lancaster.ac.uk

1 Department of Mathematics and Statistics, Lancaster University, Lancaster LA1 4YF, England 
We denote the nilradical of $L$ by $N(L)$. We define the upper nilpotent series of $L$ by

$$
N_{0}(L)=0, \quad N_{i}(L) / N_{i-1}(L)=N\left(L / N_{i-1}(L)\right) \text { for } i=1,2, \ldots
$$

The nilpotent length, $n(L)$, of $L$ is the smallest integer $n$ such that $N_{n}(L)=L$.

We define the nilpotent residual, $\gamma_{\infty}(L)$, of $L$ be the smallest ideal of $L$ such that $L / \gamma_{\infty}(L)$ is nilpotent. Clearly this is the intersection of the terms of the lower central series for $L$. Then the lower nilpotent series for $L$ is the sequence of ideals $\Gamma_{i}(L)$ of $L$ defined by $\Gamma_{0}(L)=L, \Gamma_{i+1}(L)=\gamma_{\infty}\left(\Gamma_{i}(L)\right)$ for $i \geq 0$. First we note that this series has the same length as that of the upper nilpotent series.

Lemma 1.1 Suppose that $r$ is the smallest integer such that $N_{r}(L)=L$, and that $s$ is the smallest integer such that $\Gamma_{s}(L)=0$. Then

(i) $\Gamma_{s-i}(L) \subseteq N_{i}(L)$ for $i=0, \ldots, r$;

(ii) $\quad \Gamma_{i}(L) \subseteq N_{r-i}(L)$ for $i=0, \ldots, s$; and

(iii) $r=s$.

\section{Proof}

(i) Clearly $\Gamma_{s-1}(L)$ is a nilpotent ideal of $L$ and so $\Gamma_{s-1}(L) \subseteq N_{1}(L)$. Suppose that $\Gamma_{s-k}(L) \subseteq N_{k}(L)$ for some $k \geq 1$. then

$$
\frac{\Gamma_{s-k-1}(L)+N_{k}(L)}{N_{k}(L)}
$$

is a nilpotent ideal of $L / N_{k}(L)$ and so is contained in $N_{k+1}(L) / N_{k}(L)$. Hence $\Gamma_{s-k-1}(L) \subseteq N_{k+1}(L)$, and the result follows.

(ii) Clearly $\Gamma_{1}(L) \subseteq N_{r-1}(L)$. Suppose that $\Gamma_{k}(L) \subseteq N_{r-k}(L)$ for some $k \geq 1$. Then

$$
\frac{\Gamma_{k}(L)+N_{r-k-1}(L)}{N_{r-k-1}(L)} \cong \frac{\Gamma_{k}(L)}{\Gamma_{k}(L) \cap N_{r-k-1}(L)}
$$

is nilpotent, whence $\Gamma_{k+1}(L) \subseteq N_{r-k-1}(L)$, and the result follows.

(iii) From (i) we have that $\Gamma_{s-r}(L) \subseteq N_{r}(L)$, so $s-r \geq 0$; from (ii) we see that $\Gamma_{s}(L) \subseteq$ $N_{r-s}(L)$, so $r-s \geq 0$. Thus $r=s$.

Although the upper and lower nilpotent series have equal lengths, $n$ say, we do not necessarily have that $\Gamma_{n-i}(L)=N_{i}(L)$ for $i=0, \ldots, n$, as the following example shows.

Example 1.1 Let $L$ be the metabelian Lie algebra over $\mathbb{C}$ with basis $x_{1}, x_{2}, x_{3}, x_{4}$ and nonzero products $\left[x_{1}, x_{2}\right]=x_{3},\left[x_{1}, x_{3}\right]=x_{3},\left[x_{1}, x_{4}\right]=x_{4},\left[x_{2}, x_{3}\right]=x_{4}$. Then $N_{1}(L)=$ $\mathbb{C} x_{2}+\mathbb{C} x_{3}+\mathbb{C} x_{4}, N_{2}(L)=L, \Gamma_{1}(L)=\mathbb{C} x_{3}+\mathbb{C} x_{4}, \Gamma_{2}(L)=0$.

From now on we choose to work with the upper nilpotent series. In Section 2 we investigate properties of this series, particularly its relationship to maximal subalgebras, and of Lie algebras with nilpotent length $k$ or $\leq k$. In considering factor algebras, a complication arises because, unlike the situation in group theory, there are solvable Lie algebras $L$ in which, for an ideal $I$ of $L, N(I)$ may not be contained in $N(L)$. To overcome this obstacle we introduce the notions of nilregular and strongly nilregular subalgebras of $L$; in particular, it is shown that if a maximal subalgebra of $L$ has a strongly nilregular core, its nilpotent length 
is at most one less than that of $L$. The section concludes with a fundamental decomposition theorem for Lie algebras with a given nilpotent length.

In Section 3 we introduce the class of extreme Lie algebras in which $N_{i}(L) / \phi_{i}(L)$ is a chief factor for each $i=1, \ldots, n(L)$. These are characterised in relation to the decomposition result from the previous section and described explicitly in two special cases.

The final section then considers the algebras in the title of the paper. By considering their relationship to extreme Lie algebras the minimal non- $\mathcal{N}$ Lie algebras in which every nilpotent subalgebra is abelian, and those of solvability index $\leq 3$, are characterised. The last result is that a homomorphic image of a minimal non- $\mathcal{N}$ Lie algebra is minimal non- $\mathcal{N}$ if it has a complemented minimal ideal.

Much of this is inspired by corresponding work in group theory in [5] and [4], but there are significant differences encountered in the Lie case.

\section{Properties of the Upper Nilpotent Series}

The Frattini subalgebra of $L, \phi(L)$, is the intersection of the maximal subalgebras of $L$. Since $L$ is solvable, this is an ideal of $L$ ([2, Lemma 3.4]). The Frattini series of $L$ is given by

$$
\phi_{i}(L) / N_{i-1}(L)=\phi\left(L / N_{i-1}(L)\right) \text { for } i=1,2, \ldots
$$

Lemma 2.1 Let $B$ be an ideal of $L$ with $B \subseteq \phi(L)$. Then $N_{i}(L / B)=N_{i}(L) / B$ and $\phi_{i}(L / B)=\phi_{i}(L) / B$ for every $i=1,2, \ldots, n(L)$.

Proof We have $N(L) / B=N(L / B)$, by [1, Theorem 5], and $\phi(L) / B=\phi(L / B)$, by [8, Proposition 4.3]. Suppose that $N_{k}(L) / B=N_{k}(L / B)$ and $\phi_{k}(L) / B=\phi_{k}(L / B)$. Then $B \subseteq \phi_{k+1}(L)$ and

$$
\frac{N_{k+1}(L / B)}{N_{k}(L / B)}=N\left(\frac{L / B}{N_{k}(L) / B}\right)=\frac{N_{k+1}(L) / B}{N_{k}(L) / B},
$$

whence $N_{k+1}(L) / B=N_{k+1}(L / B)$. Similarly,

$$
\frac{\phi_{k+1}(L / B)}{N_{k}(L / B)}=\phi\left(\frac{L / B}{N_{k}(L) / B}\right)=\frac{\phi_{k+1}(L) / B}{N_{k}(L) / B},
$$

which yields that $\phi_{k+1}(L) / B=\phi_{k+1}(L / B)$.

Lemma 2.2 If $A$ is an ideal of $L$ with $N_{r-1}(L) \subseteq A \subseteq N_{r}(L)$, then $n(L / A)=n(L)-r$ or $n(L)-r+1$.

Proof Put $K_{i} / A=N_{i}(L / A)$. Then it is easy to see that $K_{i} / K_{i-1}=N\left(L / K_{i-1}\right)$, and a straightforward induction argument shows that

$$
N_{r-1}(L) \subseteq A \subseteq N_{r}(L) \subseteq K_{1} \subseteq N_{r+1}(L) \subseteq \ldots \subseteq K_{n(L)-r} \subseteq N_{n(L)}(L) .
$$

If $K_{n(L)-r}=N_{n(L)}(L)$ we have $n(L / A)=n(L)-r$; otherwise, $n(L / A)=n(L)-r+1$.

Lemma 2.3 Let $M$ be a maximal subalgebra of the solvable Lie algebra L. Then

(i) $\quad N_{i}(L) \cap M \subseteq N_{i}(M)$;

(ii) $N_{i}(M)_{L} \subseteq N_{i}(L)$; 
(iii) if $N_{i}(L) \subseteq M$ then $N_{i}(M)_{L}=N_{i}(L)$;

(iv) if $k$ is the smallest positive integer such that $N_{k}(L) \nsubseteq M$ then $N_{k}(M)_{L}=N_{k}(L) \cap$ $M$; and

(v) if $N(L) \subseteq M$ then $N(M)$ acts nilpotently on $L$.

Proof (i) This is a straightforward induction proof.

(ii) It is easy to see that this holds for $i=1$. So suppose it holds for $i<k(k \geq 2)$. Then there is an $r \in \mathbb{N}$ such that $\left(N_{k}(M)_{L}\right)^{r} \subseteq N_{k}(M)^{r} \subseteq N_{k-1}(M)$, so $\left(N_{k}(M)_{L}\right)^{r} \subseteq$ $N_{k-1}(M)_{L} \subseteq N_{k-1}(L)$ by the inductive hypothesis. Thus $N_{k}(M)_{L} \subseteq N_{k}(L)$ and the result follows by induction.

(iii) This follows from (i) and (ii).

(iv) Suppose that $k$ is the smallest positive integer such that $N_{k}(L) \nsubseteq M$. Then $L=$ $N_{k}(L)+M$ and $N_{k-1}(L) \subseteq M$, so $\phi_{k}(L) \subseteq M$. Moreover, $N_{k}^{2} \subseteq \phi_{k}(L) \subseteq M$, by Lemma 2.1 and [8]. It follows that $N_{k}(L) \cap M$ is an ideal of $L$ and hence that $N_{k}(L) \cap M \subseteq N_{k}(M)_{L}$. The reverse inclusion follows as in (ii).

(v) Let $N(L) \subseteq M$. We show that $N(M)$ acts nilpotently on $L$. Suppose not, and let $L=L_{0} \dot{+} L_{1}$ be the Fitting decomposition of $L$ relative to $N(M)$. Then $L_{0}=M$ and $\left[N(L), L_{1}\right] \subseteq M \cap L_{1}=0$. Hence $L_{1} \subseteq C_{L}(N(L)) \subseteq N(L)$, giving $L=M$, a contradiction.

In general, over a field of characteristic $p>0, N(L)$ is not a characteristic ideal of $L$. The best known example is due to Jacobson and first appeared in [7]; however, it is not solvable. We shall see next that $N(L)$ is characteristic in $L$ whenever $\phi(L)$ is. First we need some lemmas.

Lemma 2.4 Let L be a Lie algebra over a field of characteristic $p>2$, let I be an abelian ideal of $L$ and let $D$ be a derivation of $L$. Then $I+D(I) \subseteq N(L)$.

Proof This follows easily from [6, Theorem 1].

Lemma 2.5 Let I be a characteristic ideal of $L$, and let $D$ be a derivation of $L$. Then $\bar{D}: L / I \rightarrow L / I: x+I \mapsto D(x)+I$ is a derivation of $L / I$.

Proof This is easy to check.

Proposition 2.6 Let $L$ be a $\phi$-free Lie algebra over a field of characteristic $p>2$. Then $N(L)$ is a characteristic ideal of $L$.

Proof Since $L$ is $\phi$-free, $N(L)=A_{1} \oplus \ldots \oplus A_{r}$, where $A_{1}, \ldots, A_{r}$ are the minimal abelian ideals of $L$, by [8, Theorem 7.4]. But $D\left(A_{i}\right) \subseteq N(L)$ for all $D \in \operatorname{Der}(L)$ and $i=1, \ldots, r$, whence the result.

Corollary 2.7 Let L be a Lie algebra over a field of characteristic $p>2$, and suppose that $\phi(L)$ is characteristic in L. Then $N(L)$ is characteristic in $L$. 
Proof This follows easily from Lemma 2.5 and Proposition 2.6.

However, there are solvable Lie algebras $L$ in which $N(L)$ is not a characteristic ideal, as the following example shows.

Example 2.1 Let $L$ be the four-dimensional Lie algebra with basis $x_{1}, x_{2}, x_{3}, x_{4}$ and nonzero products $\left[x_{4}, x_{2}\right]=x_{1},\left[x_{3}, x_{1}\right]=x_{1}$ and $\left[x_{3}, x_{2}\right]=x_{2}$ over a field $F$ of characteristic 2. Then $N(L)=F x_{1}+F x_{2}+F x_{4}$ and if $D\left(x_{1}\right)=x_{2}, D\left(x_{2}\right)=0, D\left(x_{3}\right)=0, D\left(x_{4}\right)=x_{3}$ then the extension of $D$ to $L$ by linearity is a derivation of $L$. Clearly, $D(N(L))=F x_{2}+$ $F x_{3}$. Note that $\phi(L)=F x_{1}$, so this leaves open the question of whether Proposition 2.6 holds over a field of characteristic 2 .

If we form the split extension $X=F d \dot{+} L$, where $[d, x]=D(x)$ for all $x \in L$, then $L$ is an ideal of $X$, but $N(L)$ is not.

Consequently, we shall need the following result from [12].

Proposition 2.8 Let I be a nilpotent subideal of a Lie algebra L over a field F. If $F$ has characteristic zero, or has characteristic $p$ and $L$ has no subideal with nilpotency class greater than or equal to $p-1$, then $I \subseteq N$, where $N$ is the nilradical of $L$.

Let $L$ be a Lie algebra over a field $F$ and let $U$ be a subalgebra of $L$. We call the largest integer $r$ such that $N_{r}(L) \subseteq U$ the compatibility index of $U$. As in [13], if $F$ has characteristic $p>0$, we will call $U$ nilregular if the nilradical of $U$ has nilpotency class less than $p-1$. If $U$ has compatibility index $r$, we say that $U$ is strongly nilregular if $N_{k}(U) / N_{k-1}(U)$ has nilpotency class less than $p-1$ for $k=1, \ldots, r$. If $F$ has characteristic zero we regard every subalgebra of $L$ as being nilregular. Then we have the following result.

Proposition 2.9 If I is a nilregular ideal of $L$ then $N(I) \subseteq N(L)$.

Proof This is [13, Proposition 2.1].

We shall call $L$ primitive if it has a core-free maximal subalgebra. It is said to be primitive of type 1 if it has a unique minimal ideal that is abelian; since $L$ is solvable this is the only type that can occur here (see [11]). If $B$ is an ideal of $L$ and $U / B$ is a subalgebra of $L / B$, the centraliser of $U / B$ in $L$ is $C_{L}(U / B)=\{x \in L:[x, U] \subseteq B\}$.

Proposition 2.10 Let $L$ be a solvable Lie algebra over a field $F$, and let $M$ be a maximal subalgebra of $L$ of compatibility index $r$. If $M_{L}$ is strongly nilregular, then $N_{i}(M)=$ $N_{i}\left(M_{L}\right)=N_{i}(L)$ for $i=1, \ldots, r$.

Proof First we show that if $M_{L}$ is nilregular then $N(M)=N\left(M_{L}\right)=N(L)$. We have that $N(M)$ acts nilpotently on $L$, by Lemma $2.3(\mathrm{v})$. Now $L / M_{L}$ is primitive of type 1 , and so $L / M_{L}=A / M_{L} \dot{+} M / M_{L}$, where $A / M_{L}$ is the unique minimal ideal of $L / M_{L}$ and is selfcentralising, by [11, Theorem 1.1]. Since $L=A+M$, we have that $A+N(M)$ is an ideal of $L$, and so $[A, N(M)]+M_{L}=[A, A+N(M)]+M_{L}=M_{L}$ or $A$, since $A / M_{L}$ is a chief factor of $L$. 
The former implies that $[A, N(M)] \subseteq M_{L}$, whence

$$
\frac{N(M)+M_{L}}{M_{L}} \subseteq C_{L / M_{L}}\left(\frac{A}{M_{L}}\right)=\frac{A}{M_{L}} .
$$

Thus $N(M) \subseteq A \cap M \subseteq M_{L}$.

The latter gives that $[A, N(M)]+M_{L}=A$. But then an easy induction shows that $A \subseteq A(\operatorname{ad} N(M))^{r}+M_{L}$ for every $r \in \mathbb{N}$, whence $A=M_{L}$ since $N(M)$ acts nilpotently on $L$. But this is impossible, so $N(M) \subseteq M_{L}$.

It follows that $N(M) \subseteq N\left(M_{L}\right)$ and hence $N(M) \subseteq N(L)$, by Proposition 2.9. Hence $N(M)=N\left(M_{L}\right)=N(L)$.

So suppose now that $N_{k}(M)=N_{k}\left(M_{L}\right)=N_{k}(L)$ for some $1 \leq k<r$. Then $\left(M / N_{k}(L)\right)_{L}=M_{L} / N_{k}\left(M_{L}\right)$ is nilregular, so, by the above,

$$
N\left(\frac{M}{N_{k}(M)}\right)=N\left(\frac{M}{N_{k}(L)}\right)=N\left(\frac{M_{L}}{N_{k}\left(M_{L}\right)}\right)=N\left(\frac{L}{N_{k}(L)}\right),
$$

whence

$$
\frac{N_{k+1}(M)}{N_{k}(M)}=\frac{N_{k+1}\left(M_{L}\right)}{N_{k}\left(M_{L}\right)}=\frac{N_{k+1}(L)}{N_{k}(L)}
$$

and $N_{k+1}(M)=N_{k+1}\left(M_{L}\right)=N_{k+1}(L)$. The result follows by induction.

Note that the above result is not true for all maximal subalgebras, as is shown in the next example.

Example 2.2 Let $X$ be as in Example 2.1. Then $M=F d+F x_{1}+F x_{2}$ and $L$ are both maximal subalgebras of $X$ of compatibility index 1. However, $N(L) \neq N(X)=F x_{1}+F x_{2}$, and $M_{X}=F x_{1}+F x_{2}$, so $N\left(M_{L}\right)=M_{L} \neq M=N(M)$.

Let $\mathcal{N}(k), \mathcal{N}(\leq k)$ denote the classes of Lie algebras of nilpotent length $k$ and of nilpotent length $\leq k$ respectively. Of course, over a field of characteristic zero, every Lie algebra $L \in \mathcal{N}(\leq 2)$. However, over a field of characteristic $p>0$ it is easy to construct Lie algebras $L \in \mathcal{N}(k)$ for any $k \in \mathbb{N}$.

A class $\mathcal{H}$ of finite-dimensional solvable Lie algebras is called a homomorph if $\mathcal{H}$ contains, along with an algebra $L$, all epimorphic images of $L$. A homomorph $\mathcal{H}$ is called a formation if $L / A, L / B \in \mathcal{H}$ implies that $L / A \cap B \in \mathcal{H}$, where $A, B$ are ideals of $L$. A formation $\mathcal{H}$ is said to be saturated if $L / \phi(L) \in \mathcal{H}$ implies that $L \in \mathcal{H}$.

Proposition 2.11 The class $\mathcal{N}(\leq k)$ is saturated formation for each $k \geq 1$.

Proof It is shown that $\mathcal{N}(1)$ is a saturated formation in [2, Lemma 3.7]. Suppose that it holds for $k=r$. Then $\mathcal{N}(\leq r+1)$ is clearly a homomorph. Suppose that $L / A, L / B \in$ $\mathcal{N}(\leq r+1)$. Let $S / A=N(L / A)$ and $T / B=N(L / B)$. Then $L / S, L / T \in \mathcal{N}(\leq r)$, so $L / S \cap T \in \mathcal{N}(\leq r)$. But there exist $m, n \in \mathbb{N}$ such that $S^{m} \subseteq A$ and $T^{n} \subseteq B$, so $(S \cap T)^{m+n} \subseteq A \cap B$. Hence $L / A \cap B \in \mathcal{N}(\leq r+1)$, and $\mathcal{N}(\leq r+1)$ is a formation.

Suppose now that $L / \phi(L) \in \mathcal{N}(\leq r+1)$. Then $N(L / \phi(L))=N(L) / \phi(L)$, by [8, Theorem 6.1], so $L / N(L) \in \mathcal{N}(\leq r)$ and $L \in \mathcal{N}(\leq r+1)$. It follows that $\mathcal{N}(\leq r+1)$ is saturated.

Corollary 2.12 Let $L \in \mathcal{N}(k)$ have more than one minimal ideal. Then there is at least one minimal ideal $A$ of $L$ such that $L / A \in \mathcal{N}(k)$. 
Proof If $A_{1}, \ldots, A_{n}$ are minimal ideals of $L$, where $n>1$, and $L / A_{i} \in \mathcal{N}(\leq k-1)$ for all $1 \leq k \leq n$, then $L \in \mathcal{N}(\leq k-1)$, by Proposition 2.11 .

Proposition 2.13 Let $L$ be a solvable Lie algebra over a field $F$. If $M$ is a maximal subalgebra of $L$ for which $M_{L}$ is strongly nilregular, then $n(M)=n(L)-i$ where $i \in\{0,1\}$.

Proof Let $r$ be the compatibility index of $M$. Then $L=M+N_{r+1}(L)$ and $L / N_{r+1}(L) \cong$ $M / M \cap N_{r+1}(L)=M / N_{r+1}(M)_{L}$, by Lemma 2.3 (iv). Now $N_{r}(L)=N_{r}(M)$ by Proposition 2.13, so $N_{r}(M) \subseteq N_{r+1}(M)_{L} \subseteq N_{r+1}(M)$. It follows from Lemma 2.2 that $n\left(M / N_{r+1}(M)_{L}\right)=n(M)-r-1$ or $n(M)-r$. Hence $n(L)-r-1=n(M)-r-1$ or $n(M)-r$, which gives the result.

Lemma 2.14 Let $L \in \mathcal{N}(n)$. Then we can write $L=N_{k}(L)+U_{k}$, where $U_{k}$ is a subalgebra of $L, N_{k}(L) \cap U_{k} \subseteq \phi\left(U_{k}\right)=\phi_{k+1}(L) \cap U_{k}, U_{k} \subseteq U_{k-1}$ for each $k=1, \ldots, n$. Moreover, $N\left(U_{k}\right)=N_{k+1}(L) \cap U_{k}$ for each $k=0, \ldots, n-1$.

Proof Put $U_{0}=L$. Then $L=N_{1}(L)+U_{1}$ for some subalgebra $U_{1}$ of $L$ with $N(L) \cap$ $U_{1} \subseteq \phi\left(U_{1}\right)$, by [8, Lemma 4.1]. Having constructed $U_{j}$ we construct $U_{j+1}$ such that $U_{j}=N_{j+1}(L) \cap U_{j}+U_{j+1}$ and $N_{j+1}(L) \cap U_{j+1} \subseteq \phi\left(U_{j+1}\right)$, which we can do, by using [8, Lemma 4.1] again. Now, it is easy to see inductively that $L=N_{k}(L)+U_{k}$, $N_{k}(L) \cap U_{k} \subseteq \phi\left(U_{k}\right)$ and $U_{k} \subseteq U_{k-1}$ for each $k=1, \ldots, n$. Furthermore

$$
\begin{aligned}
\frac{\phi_{k+1}(L) \cap U_{k}}{N_{k}(L) \cap U_{k}} & \cong \frac{N_{k}(L)+\phi_{k+1}(L) \cap U_{k}}{N_{k}(L)}=\frac{\phi_{k+1}(L)}{N_{k}(L)} \\
& =\phi\left(\frac{L}{N_{k}(L)}\right) \cong \phi\left(\frac{U_{k}}{N_{k}(L) \cap U_{k}}\right)=\frac{\phi\left(U_{k}\right)}{N_{k}(L) \cap U_{k}},
\end{aligned}
$$

so $\phi\left(U_{k}\right)=\phi_{k+1}(L) \cap U_{k}$.

Clearly $N_{k}(L)+N\left(U_{k}\right) \subseteq N_{k+1}(L)$ so $N\left(U_{k}\right) \subseteq N_{k+1}(L) \cap U_{k}$. But also, there is a natural number $r$ such that

$$
\left(N_{k+1}(L) \cap U_{k}\right)^{r} \subseteq N_{k}(L) \cap U_{k} \subseteq \phi\left(U_{k}\right),
$$

so $N_{k+1}(L) \cap U_{k} / \phi\left(U_{k}\right)$ is nilpotent. It follows from [8, Theorem 6.1] that $N_{k+1}(L) \cap U_{k}$ is a nilpotent ideal of $U_{k}$, so $N_{k+1}(L) \cap U_{k} \subseteq N\left(U_{k}\right)$ and equality results.

Next we have a fundamental decomposition result.

Theorem 2.15 $L \in \mathcal{N}(n)$ if and only if there are nilpotent subalgebras $B_{i}$ of $L$ for $i=$ $1, \ldots, n$ such that

$$
N_{i}(L)=B_{1}+\ldots+B_{i} \text { for } i=1, \ldots, n,
$$

(ii) $L=B_{1}+\ldots+B_{n}$,

(iii) $\left[B_{i}, B_{j}\right] \subseteq B_{i}$ for $1 \leq i \leq j \leq n$, and

(iv) $N_{i}(L) \cap U_{i} \subseteq \phi\left(U_{i}\right)=\phi_{i+1}(L) \cap U_{i}$, where $U_{i}=B_{i+1}+\ldots+B_{n}$, for $i=$ $1, \ldots, n-1$.

Proof Let $L \in \mathcal{N}(n), U_{i}$ be as in Lemma 2.14 and put $B_{i}=N\left(U_{i-1}\right)$, where $U_{0}=L$. 
(i) Clearly $B_{1}=N_{1}(L)$. Suppose that $B_{1}+\ldots+B_{k}=N_{k}(L)$. Then

$$
B_{1}+\ldots+B_{k+1}=N_{k}(L)+N\left(U_{k}\right)=N_{k}(L)+N_{k+1}(L) \cap U_{k}=N_{k+1}(L) .
$$

(ii) $B_{1}+\ldots+B_{n}=N_{n}(L)=L$.

(iii) $\left[B_{i}, B_{j}\right]=\left[N\left(U_{i}\right), N\left(U_{j}\right)\right] \subseteq\left[N\left(U_{i}\right), U_{i}\right] \subseteq N\left(U_{i}\right)=B_{i}$.

(iv) We have $U_{k}=U_{k+1}+N_{k+1}(L) \cap U_{k}=U_{k+1}+N\left(U_{k}\right)=U_{k+1}+B_{k+1}$. Hence $U_{i}=U_{i+1}+B_{i+1}=U_{i+2}+B_{i+2}+B_{i+1}=\cdots=B_{i+1}+\cdots+B_{n}$, and $N_{i}(L) \cap U_{i} \subseteq$ $\phi\left(U_{i}\right)$ from Lemma 2.14 .

The converse is clear.

\section{Extreme Lie Algebras}

The Lie algebra $L$ is monolithic if it has a unique minimal ideal $A$, the monolith of $L$. If $B$ is an ideal of $L$ and $A / B$ is a minimal ideal of $L / B$ we say that $A / B$ is a chieffactor of $L$. The series

$$
\{0\}=A_{0} \subset A_{1} \subset \ldots \subset A_{n}=L
$$

is called a chief series if $A_{i} / A_{i-1}$ is a chief factor of $L$ for each $1 \leq i \leq n$.

Lemma 3.1 Let $L$ be a Lie algebra such that $N(L) / \phi(L)$ is a chieffactor of $L$. Then $L$ has at most one complemented minimal ideal, and if $A$ is one such, then $\phi(L / A)=\phi_{2}(L) / A$.

Proof If $\phi(L)=0$, then $N(L)$ is the monolith and the result follows easily from [8, Theorems 7.3 and 7.4]. So assume that $\phi(L) \neq 0$ and let $A$ be a complemented minimal ideal of $L$. Then $A \nsubseteq \phi(L)$, so $A+\phi(L)=N(L)$. Let $M$ be a maximal subalgebra of $L$, and suppose that $\phi_{2}(L) \nsubseteq M$. Then $N(L) \nsubseteq M$, so $L=M+N(L)=M+A+\phi(L)$, giving $M+A=L$. Thus $A \nsubseteq M$. It follows that every maximal subalgebra of $L / A$ contains $\phi_{2}(L) / A$. Put $T / A=\phi(L / A)$. Then $\phi_{2}(L) \subseteq T$.

Let $M / N(L)$ be a maximal subalgebra of $L / N(L)$ and suppose that $(T+N(L)) / N(L) \nsubseteq$ $M / N(L)$. Then $M+T+N(L)=M+T=L$. But now $T \nsubseteq M$, so $\phi(L / A) \nsubseteq M$, whence $A \nsubseteq M$. It follows that $L=M+A=M$, a contradiction. Hence $\phi_{2}(L) / N(L)=$ $\phi(L / N(L)) \supseteq(T+N(L)) / N(L)$, which yields that $T=\phi_{2}(L)$.

Now suppose that $B$ is another minimal ideal of $L$ with $B \neq A$. Then

$$
B \cong(A+B) / A \subseteq N_{2}(L) / A=N(L / A),
$$

by the above. It follows that $N_{2}(L) \subseteq C_{L}(B)$. Suppose that $B \nsubseteq \phi(L)$. Then, as before, we have that $B+\phi(L)=N(L)$ and hence that $B \cong N(L) / \phi(L)$. But $C_{L}(N(L) / \phi(L))=$ $N(L)$, by [8, Theorem 7.4], since $N(L / \phi(L))=N(L) / \phi(L)$. This yields that $N(L)=$ $C_{L}(B)$, a contradiction. Hence $B \subseteq \phi(L)$ and $L$ has, at most, one complemented minimal ideal.

We call $L$ extreme if $N_{i}(L) / \phi_{i}(L)$ is a chief factor of $L$ for each $i=1,2, \ldots, n(L)$.

Lemma 3.2 Every factor algebra of an extreme Lie algebra is extreme.

Proof Let $B$ be an ideal of the extreme Lie algebra $L$. Suppose first that $B \subseteq \phi(L)$. Then $N_{i}(L / B) / \phi_{i}(L / B)$ is a chief factor of $L / B$ for each $i$, by Lemma 2.1, and $L / B$ is extreme. 
So suppose that $B \nsubseteq \phi(L)$. Then $\phi(L / B)=\phi_{2}(L) / B$, by Lemma 3.1, and the result again follows.

We say that the chief factor $A / B$ is complemented if there is a maximal subalgebra $M$ of $L$ such that $L=A+M$ and $A \cap M=B$. We define $c(L)$ to be the number of complemented chief factors in a chief series for $L$. This is independent of the particular chief series chosen, by [11, Theorem 2.3].

Let $x \in L$ and let ad $x$ be the corresponding inner derivation of $L$. If $F$ has characteristic zero suppose that $(\operatorname{ad} x)^{n}=0$ for some $n$; if $F$ has characteristic $p$ suppose that $x \in I$ where $I$ is a nilpotent ideal of $L$ of class less than $p$. Put

$$
\exp (\operatorname{ad} x)=\sum_{r=0}^{\infty} \frac{1}{r !}(\operatorname{ad} x)^{r} .
$$

Then $\exp (\operatorname{ad} x)$ is an automorphism of $L$. We call the group $\mathcal{I}(L)$ generated by all such automorphisms the group of inner automorphisms of $L$. Two subsets $U, V$ are conjugate in $L$ if $U=\alpha(V)$ for some $\alpha \in \mathcal{I}(L)$.

Then we have the following characterisation of extreme Lie algebras.

Theorem 3.3 Let L be a solvable Lie algebra. Then the following statements are equivalent:

(i) L is extreme;

(ii) $n(L)=m(L)$, the number of conjugacy classes of maximal subalgebras of $L$;

(iii) $n(L)=c(L)$; and

(iv) if $B$ is an ideal of $L$, then $L / B$ has at most one complemented minimal ideal.

Proof (i) $\Rightarrow$ (ii) $\quad$ : Let $L$ be extreme and consider the series

$$
0 \subset \phi_{1}(L) \subset N_{1}(L) \subset \ldots \subset \phi_{i}(L) \subset N_{i}(L) \subset \ldots
$$

There is a unique conjugacy class of maximal subalgebras of $L$ complementing the chief factor $N_{i}(L) / \phi_{i}(L)$ for each $i=1,2, \ldots, n(L)$, by [1]. But each maximal subalgebra of $L$ must complement one of the complemented chief factors in the above series, and must, therefore, belong to one of these $n(L)$ conjugacy classes. Hence $n(L)=m(L)$.

(ii) $\Rightarrow$ (iii) $\quad$ : We use induction on the dimension of $L$. Suppose that $L$ is a Lie algebra satisfying $n(L)=m(L)$ and assume that the implication holds for Lie algebras of smaller dimension than that of $L$. If $\phi(L) \neq 0$, we have $n(L / \phi(L))=$ $n(L)=m(L)=m(L / \phi(L)$, and so, by induction, $n(L)=n(L / \phi(L))=$ $m(L / \phi(L))=c(L / \phi(L))=c(L)$.

So suppose that $\phi(L)=0$. Then $N(L)=A \operatorname{soc}(L)$ and each of the $r$ (say) minimal ideals in $A \operatorname{soc}(L)$ is complemented, by [8, Theorem 7.4 and Lemma 7.2]. It follows that $n(L)=m(L) \geq m(L / N(L))+r \geq n(L)-1+r$. Hence $r=1$ and, by induction, $c(L)=1+c(L / N(L))=1+n(L / N(L))=n(L)$.

(iii) $\Rightarrow$ (i) $\quad$ : This follows from the fact that there is at least one complemented chief factor $A / B$ satisfying $\phi_{i}(L) \leq B<A \leq N_{i}(L)$ for each $i=1,2, \ldots, n(L)$.

(i) $\Leftrightarrow$ (iv) : If $L$ is extreme, then so is $L / B$, by Lemma 3.2. Hence $L / B$ has at most one complemented minimal ideal, by Lemma 3.1 . 
Conversely, suppose that $L$ satisfies (iv) and consider $L / \phi_{i}(L)$. Since $N_{i}(L) / \phi_{i}(L)$ is the direct sum of complemented minimal ideals of $L / \phi_{i}(L)$, as above, it follows that $N_{i}(L) / \phi_{i}(L)$ is a chief factor of $L / \phi_{i}(L)$. Hence $L$ is extreme.

Lemma 3.4 Let $L$ be an extreme Lie algebra.

(i) If $L$ is nilpotent, then $\operatorname{dim} L=1$.

(ii) If $L \in \mathcal{N}(n)$ then $N_{n-1}(L)=\Gamma_{1}(L)=L^{k}$ has codimension one in $L$, for all $k \geq 2$.

Proof (i) Since $L$ is nilpotent, $\phi(L)=L^{2}$, and so $\operatorname{dim} L / L^{2}=1$. The result follows. (ii) This follows from Lemma 3.2 and (i).

Theorem 3.5 The Lie algebra $L \in \mathcal{N}(n)$ is extreme if and only if $L$ has the decomposition given in Theorem 2.15, $\operatorname{dim} B_{n}=1$ and $N\left(U_{k}\right) / \phi\left(U_{k}\right)$ is a chief factor of $U_{k}$ for each $k=0, \ldots, n-1$.

Proof We have that

$$
\begin{aligned}
\frac{N_{k+1}(L)}{\phi_{k+1}(L)} & =\frac{N_{k+1}(L) \cap U_{k}+N_{k}(L)}{\phi_{k+1}(L) \cap U_{k}+N_{k}(L)} \cong \frac{\left(N_{k+1}(L) \cap U_{k}+N_{k}(L)\right) / N_{k}(L)}{\left(\phi_{k+1}(L) \cap U_{k}+N_{k}(L)\right) / N_{k}(L)} \\
& \cong \frac{N_{k+1}(L) \cap U_{k} / N_{k}(L) \cap U_{k}}{\phi_{k+1}(L) \cap U_{k} / N_{k}(L) \cap U_{k}} \cong \frac{N_{k+1}(L) \cap U_{k}}{\phi_{k+1}(L) \cap U_{k}}=\frac{N\left(U_{k}\right)}{\phi\left(U_{k}\right)} .
\end{aligned}
$$

Also $\operatorname{dim} B_{n}=1$ by Lemma 3.4 (ii).

If $S$ is a subalgebra of $L$, we will denote by $\bar{S}$ the image of $S$ under the canonical homomorphism from $L$ onto $L / \phi(L)$. We have the following characterisation of those Lie algebras $L \in \mathcal{N}(\leq 2)$ that are extreme, which includes all extreme Lie algebras over a field of characteristic zero.

Corollary 3.6 Let $L \in \mathcal{N}(\leq 2)$. Then $L$ is extreme if and only if one of the following holds.

(i) $\operatorname{dim} L=1$; or

(ii) $\bar{L}=\bar{A} \dot{+} \bar{U}$ where $\bar{A}=\overline{N(L)}$ is the monolith of $\bar{L}$ and $\bar{U}$ is a one-dimensional subalgebra of $\bar{L}$ which acts irreducibly on $\bar{A}$.

Proof This is just the cases $n=1,2$ in Theorem 3.5.

Corollary 3.7 Let L be supersolvable. Then $L$ is extreme if and only if one of the following holds.

(i) $\operatorname{dim} L=1$; or

(ii) $L / \phi(L)$ is the two-dimensional non-abelian Lie algebra.

Proof Let $L$ be supersolvable and extreme. Then $\operatorname{dim} \overline{N(L)}=1$ and so $\operatorname{dim} \bar{L} / C_{\bar{L}}(\overline{N(L)})=1$. But $\overline{N(L)}=N(\bar{L})$, so $C_{\bar{L}}(\overline{N(L)}) \subseteq \overline{N(L)}$. It follows that 
$\operatorname{dim}(L / N(L)) \leq 1$ and $L \in \mathcal{N}(\leq 2)$. But now either $\operatorname{dim} L=1$ or $\bar{L}=\bar{A} \dot{+} \bar{U}$ where $\operatorname{dim} \bar{A}=\operatorname{dim} \bar{U}=1$, by Theorem 3.5. In the latter case $\operatorname{dim} \bar{L}=2$ and $\bar{L}$ cannot be abelian.

Conversely, if $\operatorname{dim}(L / \phi(L)) \leq 2$ then $L / \phi(L)$ is supersolvable, and so $L$ is supersolvable, by [1, Theorem 6]. Clearly $L$ is also extreme in each of cases (i) and (ii).

Example 3.1 It is easy to check that every three-dimensional Lie algebra as described in Corollary 3.7 has a basis $x, y, z$ with non-zero products $[x, y]=y+z,[x, z]=\alpha z$ for some $0 \neq \alpha \in F$. Moreover, no two of these with different values of $\alpha$ are isomorphic.

\section{Minimal Non- $\mathcal{N}$ Algebras}

If $\mathcal{X}$ is a class of Lie algebras, we say that $L$ is minimal non- $\mathcal{X}$ if every proper subalgebra of $L$, but not $L$ itself, belongs to $\mathcal{X}$. We say that $L$ is minimal non- $\mathcal{N}$ if it is minimal non$\mathcal{N}(\leq k)$ for some $k$; in other words, if its nilpotent length is greater than that of any of its proper subalgebras. Over a field of characteristic zero a Lie algebra can only be minimal non- $\mathcal{N}(1)$ and these are described in [9].

Lemma 4.1 Let $L$ be minimal non- $\mathcal{N}(\leq k-1)$ and let $M$ be a maximal subalgebra of $L$. Then

(i) $L^{2}=N_{k-1}(L)$ has codimension one in $L$;

(ii) if $N_{i}(M) \nsubseteq N_{k-1}(L)$ then $N_{k-1}(L) \cap N_{i}(M)$ has codimension one in $N_{i}(M)$ for $i=1, \ldots, k-1$; and

(iii) if $N(L) \subseteq M$ then $N(M) \subseteq N_{k-1}(L)$.

Proof (i) Let $M$ be a maximal subalgebra of $L$ containing $N_{k-1}(L)$. Since $L / N_{k-1}(L)$ is nilpotent, $M$ is an ideal of $L$ and has codimension one in $L$. But $N_{k-1}(M)_{L}=$ $N_{k-1}(L)$, by Lemma 2.3 (ii), so, if $N_{k-1}(L) \neq M$, then $M$ has nilpotent length $k$, contradicting the fact that it is minimal non- $\mathcal{N}(k)$. Hence $M=N_{k-1}(L)$.

Now let $M$ be any maximal subalgebra containing $L^{2}$, so $M$ is an ideal of codimension one in $L$. We have $M \cap N_{i}(L)=N_{i}(M)_{L}$ for each $i=1, \ldots k-1$ by Lemma 2.3 (i) and (ii). It follows that $M \cap N_{k-1}(L)=N_{k-1}(M)_{L}=M$, so $M \subseteq N_{k-1}(L)$, whence $M=N_{k-1}(L)=L^{2}$.

(ii) Suppose $N_{i}(M) \nsubseteq N_{k-1}(L)$. Then $L=N_{k-1}(L)+N_{i}(M)$, so

$$
\frac{L}{N_{k-1}(L)} \cong \frac{N_{i}(M)}{N_{k-1}(L) \cap N_{i}(M)},
$$

whence the result.

(iii) Let $N(L) \subseteq M$. Then $N(M)$ acts nilpotently on $L$, by Lemma 2.3 (v). If $N(M) \nsubseteq$ $N_{k-1}(L)$ then $L=N_{k-1}(L)+N(M)$ and $L / N_{k-2}(L)$ is nilpotent, a contradiction.

In group theory, every minimal non- $\mathcal{N}(k)$ group is extreme, and so a natural question is whether this holds for Lie algebras. We show next that this is 'usually' the case for Lie algebras. We call a class $\mathcal{H}$ of Lie algebras a semi-homomorph if, for all $L \in \mathcal{H}$,

(i) $L / N(L) \in \mathcal{H}$; and

(ii) if $A$ is an ideal of $L$ and $A \subseteq \phi(L)$, then $L / A \in \mathcal{H}$. 
Lemma 4.2 Let $\mathcal{H}$ be the class of Lie algebras $L$ in which all maximal subalgebras of $L$ have strongly nilregular cores. Then $\mathcal{H}$ is a semi-homomorph.

Proof (i) Let $M / N(L)$ be a maximal subalgebra of $L / N(L)$. Clearly we have that $(M / N(L))_{L / N(L)}=M_{L} / N(L)$ and $M_{L} / N(L)$ has compatibility index one less than that of $M_{L}, r-1$ say. Then

$$
\frac{N_{i}\left(M_{L} / N(L)\right)}{N_{i-1}\left(M_{L} / N(L)\right)} \cong \frac{N_{i+1}\left(M_{L}\right)}{N_{i}\left(M_{L}\right)},
$$

which has nilpotency class $<p-1$ for $i=1, \ldots, r-1$, since $M_{L}$ is strongly nilregular. Hence $M / N(L)$ has a strongly nilregular core.

(ii) Let $M / A$ be a maximal subalgebra of $L / A$. Then $(M / A)_{L / A}=M_{L} / A$. Also, $N_{i}(L / A)=N_{i}(L) / A$, by Lemma 2.1 , so $M_{L} / A$ has the same compatibility index as $M_{L}, r$ say. Also $N_{i}(M)=N_{i}\left(M_{L}\right)=N_{i}(L)$ for $i=1, \ldots, r$, by Proposition 2.10.

We claim that $N_{i}\left(M_{L} / A\right)=N_{i}\left(M_{L}\right) / A$ for each $i=1, \ldots, n\left(M_{L}\right)$. Put $K_{i} / A=$ $N_{i}\left(M_{L} / A\right)$, so $K_{i+1} / K_{i}=N\left(M_{L} / K_{i}\right)$. Then $K_{1}$ is a subideal of $L, A \subseteq K_{1} \cap \phi(L)$ and $K_{1} / A$ is nilpotent, so $K_{1}$ is nilpotent, by [8, Theorem 6.1]. It follows that $K_{1} \subseteq$ $N\left(M_{L}\right)$ and $N\left(M_{L} / A\right)=N\left(M_{L}\right) / A$. Hence

$$
K_{1} \subseteq N\left(M_{L}\right) \subseteq K_{2} \subseteq \ldots \subseteq K_{i} \subseteq N_{i}\left(M_{L}\right) \subseteq K_{i+1} \subseteq N_{i+1}(L) \subseteq \ldots
$$

and $K_{i+1} / K_{i} \subseteq N_{i+1}(L) / K_{i}=N_{i+1}\left(M_{L}\right) / K_{i}$ for $i=1, \ldots, r-1$. It follows that $N_{i+1}\left(M_{L} / A\right)=K_{i+1} / A \subseteq N_{i+1}(L) / A$. The reverse inclusion is clear and the claim is established.

Now we have that

$$
\frac{N_{i}\left(M_{L} / N(L)\right)}{N_{i-1}\left(M_{L} / N(L)\right)} \cong \frac{N_{i}\left(M_{L}\right)}{N_{i-1}\left(M_{L}\right)},
$$

which has nilpotency class $<p-1$ for $i=1, \ldots, r$, since $M_{L}$ is strongly nilregular. Hence $M / N(L)$ has a strongly nilregular core.

A solvable primitive algebra has a unique minimal, self-centralising, ideal $A$ such that $L=A \dot{+} U$ (see [11]). We shall say that a class of Lie algebras $\mathcal{H}$ has the primitive quotient property if, for every primitive algebra $L$ in $\mathcal{H}$ with minimal ideal $A, L / A$ is minimal non- $\mathcal{N}$.

Theorem 4.3 Let $\mathcal{H}$ be a semi-homomorph with the primitive quotient property, and let $L \in \mathcal{H}$ be a Lie algebra which is minimal non- $\mathcal{N}(\leq n)$. Then

(i) L is extreme; and

(ii) $L / N(L)$ is minimal non- $\mathcal{N}(\leq n-1)$.

Proof (i) We use induction on $\operatorname{dim} L$. Suppose first that $\phi(L) \neq 0$. Let $A$ be a minimal ideal of $L$ contained in $\phi(L)$. Then $n(L / A)=n(L)$, so $L / A$ is minimal non- $\mathcal{N}$. By the inductive hypothesis, $L / A$, and hence $L$ is extreme. So suppose that $\phi(L)=0$. If there are at least two minimal ideals then there is at least one, $A$ say, such that $n(L / A)=n(L)$, by Corollary 2.12. But then $L=A \dot{+} M$ for some maximal subalgebra $M$ of $L$, and $n(M)=n(L / A)=n(L)$, a contradiction. 
Thus there is a unique minimal ideal $A=N(L), L$ is primitive and $n(L / A)=$ $n(L)-1$. Since $\mathcal{H}$ has the primitive quotient property, $L / A$ is minimal non- $\mathcal{N}$. Moreover, since $\mathcal{H}$ is a semi-homomorph, $L / A$, and thus $L$, is extreme, by induction.

(ii) Consider the series

$$
0 \subset \phi_{1}(L) \subset N_{1}(L) \subset \ldots \subset \phi_{i}(L) \subset N_{i}(L) \subset \ldots,
$$

and let $M$ be a maximal subalgebra of $L$ containing $N_{1}(L)$. Then $M$ must complement one of the complemented chief factors $N_{k}(L) / \phi_{k}(L)$ for some $2 \leq k \leq n$ in the above series. But then $L=N_{k}(L)+M, M \cap N_{k}(L)=\phi_{k}(L)$ and $N_{i}(M)=N_{i}(L)$ for $i=$ $1, \ldots, k-1$, by Lemma 2.3. Thus $n(M)-k+1=n\left(M / N_{k-1}(L)\right)=n\left(L / N_{k}(L)\right)=$ $n(L)-k$, whence $n\left(M / N_{1}(L)\right)=n(M)-1=n(L)-2=n\left(L / N_{1}(L)\right)-1$ and $L / N_{1}(L)$ is minimal non- $\mathcal{N}(n-1)$.

Corollary 4.4 Let $L$ be a Lie algebra in which all maximal subalgebras have strongly nilregular cores and which is minimal non $\mathcal{N}(\leq n)$. Then $L$ is extreme and $L / N(L)$ is minimal non- $\mathcal{N}(\leq n-1)$.

Proof Let $\mathcal{H}$ be the class of Lie algebras whose maximal subalgebras have strongly nilregular cores. Then $\mathcal{H}$ is a semi-homomorph, by Lemma 4.2. Let $L$ be a primitive algebra in $\mathcal{H}$ with minimal ideal $A=N(L)$, and let $M$ be a maximal subalgebra containing $A$. Then $N(M)=A$ by Proposition 2.10 , so $L / A$ is minimal non- $\mathcal{N}(\leq n-1)$ and $\mathcal{H}$ satisfies the primitive quotient property. The result now follows from Theorem 4.3.

A Lie algebra $L$ is called an $A$-algebra if all of its nilpotent subalgebras are abelian. These arise in the study of constant Yang-Mills potentials and in relation to the problem of describing residually finite varieties. The structure of solvable Lie $A$-algebras was studied in some detail in [10]. In the case of an $A$-algebra the lower nilpotent series and the derived series coincide ([10, Lemma 2.3]), and so the terms "derived length" and "nilpotent length" are identical.

Corollary 4.5 If $L$ is an A-algebra which is minimal non- $\mathcal{N}(\leq n)$, then $L$ is extreme and $L / N(L)$ is minimal non $-\mathcal{N}(\leq n-1)$.

Proof Let $\mathcal{H}$ be the class of $A$-algebras. Then $\mathcal{H}$ is a semi-homomorph, by [10, Lemma 2.1 (iii)]. Let $L \in \mathcal{H}$ be primitive with minimal ideal $A=N(L)$ and let $M$ be a maximal subalgebra containing $A$. Then $N(M)$ is abelian and so $[N(M), A]=0$, giving $N(M) \subseteq$ $C_{L}(A)=A$. It follows that $N(M)=A$ and so $\mathcal{H}$ has the primitive quotient property.

Corollary 4.6 Let $L$ be minimal non $\mathcal{N}(\leq 2)$ and have solvability index $\leq 3$. Then $L$ is extreme and $L / N(L)$ is minimal non- $\mathcal{N}(\leq 1)$.

Proof Let $\mathcal{H}$ be the class of Lie algebras of solvability index $\leq 3$. Then $\mathcal{H}$ is clearly a semi-homomorph. Let $L \in \mathcal{H}$ be primitive with minimal ideal $A=N(L)$ and let $M$ be a maximal subalgebra containing $A$. If $L$ has solvability index $\leq 2$ it is clear that $L / A$ is minimal non- $\mathcal{N}$, so assume that $L$ has index 3 . 
We have $L^{(1)}=N_{2}(L)$, by Lemma 4.1 (i) and $L^{(2)} \subseteq N(L)=A$, so $N_{2}(L) / A$ is abelian. Now $N(M) \subseteq N_{2}(L)$ by Lemma 4.1 (iii), and so

$$
\left[N_{2}(L), N(M)\right] \subseteq N_{2}(L)^{2} \subseteq A \subseteq M .
$$

Then either $M \neq N_{2}(L)$ or $L=N_{2}(L)+M$, in which case $N(M)$ is an ideal of $L$ and $N(M)=A$. In either case $M / A$ is nilpotent and so $\mathcal{H}$ has the primitive quotient property.

Example 4.1 Note that there are extreme Lie algebras which are not minimal non- $\mathcal{N}$. For example, let $L$ be the Lie algebra over any field $F$ with basis $x, y, z$ with non-zero products $[x, y]=y+z,[x, z]=z$. Then $\phi(L)=F z$ and $N(L)=F y+F z$, so $L$ is extreme. However, if $M=F x+F z$ then $n(M)=2=n(L)$.

Next we seek to characterise the algebras considered in Corollaries 4.5 and 4.6. We can characterise the $A$-algebras that are also minimal non- $\mathcal{N}$ as follows.

Theorem 4.7 Let L be a Lie A-algebra of derived length $n+1$ over a field $F$. Then $L$ is minimal non- $\mathcal{N}$ if and only if the following hold.

(i) $L=A_{n} \dot{+} A_{n-1} \dot{+} \ldots \dot{+} A_{1} \dot{+} F x$ where $A_{i}$ is an abelian subalgebra of $L$ for each $1 \leq i \leq n$;

(ii) $L^{(\bar{i})}=A_{n} \dot{+} A_{n-1} \dot{+} \ldots \dot{+} A_{i}$ for each $1 \leq i \leq n$;

(iii) $\left[A_{i}, A_{j}\right] \subseteq A_{j}$ for $j>i$;

(iv) $A_{i}$ is an irreducible $L / L^{(i+1)}$-module for each $0 \leq i \leq n$; and

(v) $N_{n-i+1}(L)=L^{(i)}$ for each $0 \leq i \leq n$.

Proof Suppose first that $L$ is minimal non- $\mathcal{N}$. Since $L$ is an $A$-algebra of derived length $n+1, L=A_{n} \dot{+} A_{n-1} \dot{+} \ldots \dot{+} A_{1} \dot{+} A_{0}$ and $L^{(i)}=A_{n} \dot{+} A_{n-1} \dot{+} \ldots \dot{+} A_{i}$, where $A_{i}$ is an abelian subalgebra of $L$ for each $0 \leq i \leq n$, by [10, Corollary 3.2]. But $\operatorname{dim} A_{0}=1$, by Lemma 4.1 (i), so $A_{0}=F x$ for some $x \in L$. This gives (i) and (ii). The decomposition in (i) follows from the splitting of a Lie $A$-algebra over each term in its derived series ([10, Theorem 3.1]), so $L=A_{n} \dot{+} B_{n}$, where $A_{n}=L^{(n)}, B_{n}=A_{n-1} \dot{+} B_{n-1}$, where $A_{n-1}=B_{n}^{(n-1)}$, and so on. But now $\left[A_{i}, A_{j}\right] \subseteq L^{(j)} \cap B_{j+1} \subseteq A_{j}$ if $j>i$, giving (iii).

A straightforward induction argument shows that $A_{i} \subseteq N_{n-i-1}$ for $0 \leq i \leq n$. We now establish (iv) and (v) by induction on $n$. Then (iv) clearly holds for $i=0$, and (v) holds for $i=0$ by Lemma 4.1 (i), so suppose that they hold for all $i \geq k(k \geq 0)$. Then $N_{n-k+1}(L)=L^{(k)}=A_{n} \dot{+} \ldots \dot{+} A_{k}$ and $L^{(k+1)}=A_{n} \dot{+} \ldots \dot{+} A_{k+1} \subseteq N_{n-k}(L)$. It follows that $N_{n-k}(L)=L^{(k+1)}$ by the irreducibility of $A_{k}$ and the fact that $N_{n-k}(L) \neq N_{n-k+1}(L)$ (since $L$ has nilpotent length $n+1$ ).

Also $\phi_{n-k}(L) \subseteq L^{(k+1)}=N_{n-k}(L)$, and $N_{n-k}(L) / \phi_{n-k}(L)$ is irreducible, since $L$ is extreme, by Theorem 4.3. Hence $M=\phi_{n-k}(L) \dot{+} A_{k} \dot{+} \ldots \dot{+} A_{1} \dot{+} F x$ is a maximal subalgebra of

$$
A_{k+1} \dot{+} A_{k} \dot{+} \ldots \dot{+} A_{1} \dot{+} F x \cong L / L^{(k+2)}=L / N_{n-k-1}(L) .
$$

But $L / N_{n-k-1}(L)$ is minimal non- $\mathcal{N}(k+1)$, by Theorem 4.3 (ii), so $M \in \mathcal{N}(k+1)$. It follows that $N(M)=\phi_{n-k}(L) \dot{+} A_{k}$, so $\left[\phi_{n-k}(L), A_{k}\right]=0$. But $A_{k}$ is a Cartan subalgebra of $A_{k+1} \dot{+} A_{k}$, by [10, Theorem 3.1], so $\phi_{n-k}(L)=0$ and $A_{k}$ is an irreducible $L / L^{(k+1)}$ module. This establishes (iv) and (v). 
Conversely, suppose that (i)-(v) hold and let $M$ be a maximal subalgebra of $L$. Clearly $L \in \mathcal{N}(n+1)$. Let $i$ be the smallest integer such that $L^{(i)} \nsubseteq M$. Then $L^{(i)} / L^{(i+1)}$ is a minimal ideal of $L / L^{(i+1)}$, so $M / L^{(i+1)}$ complements $L^{(i)} / L^{(i+1)}$ in $L / L^{(i+1)}$. Hence $M^{(i)} \subseteq M \cap L^{(i)} \subseteq L^{(i+1)}$. But $n\left(L^{(i+1)}\right)<n-i+1$ by (v). Hence $n(M)<n+1$ and $L$ is minimal non- $\mathcal{N}$.

Recall the following result from [3].

Theorem 4.8 ([3, Theorem 4]) Let $L$ be solvable and $\phi$-free. Then $L$ is minimal non(nilpotent-by-abelian) if and only if $F$ has characteristic $p>0$ and $L=A \dot{+} B$, where $A$ is the unique minimal ideal of $L, \operatorname{dim} A \geq 2, A^{2}=0$, and either $B=M \dot{+} F x$, where $M$ is a minimal ideal of $B$ such that $M^{2}=0$ (type I), or $B$ is the three-dimensional Heisenberg algebra (type II). Moreover, if $p \geq 3$ then $\operatorname{dim} A$ is divisible by $p$.

Then we have the following characterisation of the algebras of solvability index 3 which are minimal non- $\mathcal{N}(2)$.

Theorem 4.9 Let L be a solvable Lie algebra of solvability index 3. Then $L$ is minimal non- $\mathcal{N}(2)$ if and only if it is minimal non-(nilpotent-by-abelian) of type $I$.

Proof Let $L$ be minimal non- $\mathcal{N}(2)$, and denote the image of a subalgebra $S$ of $L$ under the natural homomorphism onto $L / \phi(L)$ by $\bar{L}$. Then $\bar{L}=\overline{N(L)} \dot{+} \bar{U}$ where $U$ is a subalgebra of $L$, by [8, Theorems 7.3 and 7.4]. Moreover, $\bar{U}=\bar{A} \dot{+} F \bar{x}$ where $\bar{A}$ is abelian, $N_{2}(L)=$ $N(L)+A$ and $L / N(L)$ is minimal non-nilpotent, by Corollary 4.6. It follows from [9, Theorem 2.1] that $N_{2}(L) / N(L)$ is irreducible. Now $U$ is a maximal subalgebra of $L$, so $U \in \mathcal{N}(2)$, which yields that $N(U)=A$ and $U$ is nilpotent-by-abelian.

Let $M$ be any maximal subalgebra of $L$. Then either $M \cong U$ or $N(L) \subseteq M$. Suppose that $N(L) \subseteq M$. Then $M=N_{2}(L)$ or $L=N(L)+F x$. In either case $M$ is nilpotentby-abelian. Hence $L$ is minimal non-(nilpotent-by-abelian). It is of type I, since otherwise $L \in \mathcal{N}(2)$.

Conversely, suppose that $L$ is minimal non-(nilpotent-by-abelian) of type I. Then $L$ has solvability index 3 and the maximal subalgebras of $L$ are nilpotent-by-abelian, as in the paragraph above. Clearly $L$ itself is not nilpotent-by-abelian.

Lie algebras as described in Theorem 4.9 do exist over every field of characteristic $p>0$, as is shown in [3]; over an algebraically closed field they are minimal non-supersolvable ([3, Theorem 5]). Finally we show that a homomorphic image of a minimal non- $\mathcal{N}$ Lie algebra is minimal non- $\mathcal{N}$ if it has a complemented minimal ideal.

Theorem 4.10 If $L$ is a minimal non- $\mathcal{N}$ Lie algebra in which all maximal subalgebras have nilregular cores, and $A / B$ is a complemented chief factor of $L$, then $L / B$ is minimal non- $\mathcal{N}$.

Proof Suppose there is a chief series of $L$ through $A$ and $B$ in which $A / B$ is the $k$ th complemented factor, where $1 \leq k \leq c(L)$. If $k=1$, then $B \subseteq \phi(L)$ and so $n(L / B)=n(L)$, in which case $L / B$ is minimal non- $\mathcal{N}$ because $L$ is. 
So let $k>1$ and assume that the theorem holds for the $(k-1)$ th complemented factor $C / D$. Without loss of generality we may assume that $D=0$. Then $A / B$ is the second complemented factor in some chief series of $L$. It follows from Theorem 4.3 that $L$ and $L / N(L)$ are extreme, and so every chief series of $L$ has only one complemented chief factor below $N(L)$, by Theorem 3.3. If $N(L) \cap A \nsubseteq B$ then $N(L) \cap A / N(L) \cap B$ is a complemented chief factor of $L$ and $L$ would have a chief series with two complemented chief factors below $N(L)$, a contradiction. Hence $N(L) \cap A \subseteq B$.

Let $n=n(L)$. Then $L / A$ and $L / B$ are both extreme, by Lemma 3.2 and so

$$
n(L / B)=c(L / B)=n-1 \text { and } n(L / A)=c(L / A)=n-2,
$$

by Theorem 3.3. Let $M / B$ be a maximal subalgebra of $L / B$. We have $B \nsubseteq \phi(L)$ and $N(L) / \phi(L)$ is a chief factor of $L$, so $M \supseteq \phi(L)+B \supseteq N(L)$. But $L / N(L)$ is minimal non$\mathcal{N}$, by Theorem 4.3, so $n(M / N(L)) \leq n-2$. It follows that $N_{n-2}(M) \subseteq N(L) \cap A \subseteq B$, whence $n(M / B) \leq n-2<n(L / B)$ and $L / B$ is minimal non- $\mathcal{N}$, as claimed.

Open Access This article is distributed under the terms of the Creative Commons Attribution 4.0 International License (http://creativecommons.org/licenses/by/4.0/), which permits unrestricted use, distribution, and reproduction in any medium, provided you give appropriate credit to the original author(s) and the source, provide a link to the Creative Commons license, and indicate if changes were made.

\section{References}

1. Barnes, D.W.: On the cohomology of soluble Lie algebras. Math. Zeit. 101, 343-49 (1967)

2. Barnes, D.W., Gastineau-Hills, H.M.: On the theory of soluble Lie algebras. Math. Z. 106, 343-354 (1968)

3. Bowman, K., Towers, D.A.: On almost Nilpotent-by-Abelian Lie Algebras. Lin. Alg. Appl. 247, 159-167 (1996)

4. Carter, R., Fischer, B., Hawkes, T.: Extreme classes of finite soluble groups. J. Algebra 9, 285-313 (1968)

5. Frick, M.: The nilpotent length of finite soluble groups. J. Austral. Math. Soc. 16, 357-362 (1973)

6. Maksimenko, D.V.: On action of outer derivations on nilpotent ideals of Lie algebras. Algebra Discrete Math. 1, 74-82 (2009)

7. Seligman, G.B.: Characteristic ideals and the structure of Lie algebras. Proc. Amer. Math. Soc. 8(1), 159-164 (1957)

8. Towers, D.A.: A Frattini theory for algebras. Proc. London Math. Soc. (3) 27, 440-462 (1973)

9. Towers, D.A.: Lie algebras all of whose proper subalgebras are nilpotent. Lin Alg. Appl. 32, 61-73 (1980)

10. Towers, D.A.: Solvable Lie A-algebras. J. Algebra 340, 1-12 (2011)

11. Towers, D.A.: Maximal subalgebras and chief factors of Lie algebras. J. Pure Appl. Algebra 220, 482493 (2016)

12. Towers, D.A.: On n-maximal subalgebras of Lie algebras. Proc. Amer. Math. Soc. 144, 1457-1466 (2016)

13. Towers, D.A.: The generalised nilradical of a Lie algebra. J. Algebra 470, 197-218 (2017) 